\title{
INTELLIGENT METHOD OF ELECTRIC DRIVE DIAGNOSTIC WITH DUE ACCOUNT FOR ITS OPERATION MODE
}

\author{
Alexey Grigoryevich Bulgakov ${ }^{*}$ Tatyana Nikolaevna Kruglova ${ }^{2}$ \\ ${ }^{1}$ Southwest State University, Kursk, Russia \\ ${ }^{2}$ South-Russian State Polytechnic University, Novocherkassk, Rusia
}

In this article is proposed an intelligent method for diagnosing a technical condition, which makes it possible to distinguish a true malfunction of object from changing the parameters of its operating mode. As a result of numerous experiments has been revealed the dependence of measurement of wavelet transformation coefficients on the characteristic scales of a serviceable and faulty engine under different loading regimes. On the basis of the received information has been developed a neural classification network which makes it possible to reveal the current state of the object. Further studies have shown that any parent wavelet can be used to imple-ment the proposed method. The study of the state of the drive under various loads confirms the correctness of the theoretical calculations and the adequacy of the model.

Key words: Diagnosis of the electric drive, Neural network method, Wavelet transformation, Operating mode of the drive

\section{INTRODUCTION}

In the process of long-term operation of electrical equipment can arise various kinds of defects, which lead to long idle periods and inevitable financial losses. One of the ways to solve the problem of sudden equipment failures is to monitor and evaluate its current status, identified by means of diagnostic methods and tools, allowing to classify the current state of the object to one of the predetermined classes of diagnoses and to determine the locations of the defects that have arisen. To implement these methods, it is advisable to select a functional approach that allows you to identify the state of the object without decommissioning it. The selected diagnostic parameters should identify all the object faults that have occurred, be simple to measure and analyze. These requirements are met by vibration, noise and current consumption. The traditional method of analyzing such parameters is the Fourier transform [01], which has a number of significant drawbacks [02], which do not allow its use for automatic diagnostics of electric drives operating under dynamic loads. Therefore, the problem of synthesizing the method of diagnosis, which allows to determine with a high degree of accuracy the current state of the object, distinguishing the load change from a malfunction and to find all the defects that have arisen, is very relevant.

\section{STATEMENT OF THE RESEARCH TASK}

The purpose of this study is to develop a method for analyzing signals, which allows finding a fault with a high degree of certainty and distinguishing it from changing the operating mode of the drive.

To solve this problem, it is supposed to apply wavelet analysis of signals, which considers the analyzed time functions in terms of oscillations localized in time and frequency, providing a two-dimensional sweep of one-dimensional signals. In this case, the frequency and coordinate are considered as independent variables, which makes it possible to analyze the signals in two spaces at once. The wavelet functions of the basis allow us to concentrate our attention on certain local features of the analyzed processes, which can not be detected using traditional Fourier and Laplace transforms.

The wavelet transformation of a signal is its representation in the form of a generalized series or the Fourier integral over a system of basis functions [03-05]

$$
\psi_{a b}(t)=\frac{1}{\sqrt{a}} \psi\left(\frac{t-b}{a}\right)
$$

constructed from the parent (original) wavelet $\psi(t)$ possessing certain properties, due to the time shift operations $b$ and the time scale change a. The factor $1 / \sqrt{a}$ ensures that the norm of these functions is independent of the scaling number $a$. Small values, a correspond to small scales $\psi_{a b}(t)$, or high frequencies ( $\left.\omega \sim 1 / a\right)$, large parameters a - to large scale $\psi_{a b}(t)$, i.e. stretching the parent wavelet $\psi(t)$ and compressing its spectrum. Thus, the wavelet scale, as a unit of the time-frequency representation of the signals, is inversely proportional to the frequency (Figure 1).

At the present time has been accumulated a considerable amount of knowledge to trouble-shoot of electrical equipment using Fourier transforms, and have been calculated the characteristic frequencies for all major faults. The recalculation of the Fourier frequencies of the spectrum into the scale of the wavelet, according to the dependence in Figure 1, will allow the analysis of signals into wavelet space [06] and find new signal characteristics that can not be detected using Fourier analysis. 
It is assumed that the use of the wavelet transformation will allow a more accurate identification of the state of the electric drive, distinguishing the faulty state from a change in load, and to reveal all its faults. For this, it is necessary to carry out a series of experimental studies, based on the results of the analysis of which it is planned to develop a model for diagnosing the technical condition, which makes it possible to distinguish a faulty state of an object from a change in its operating mode.

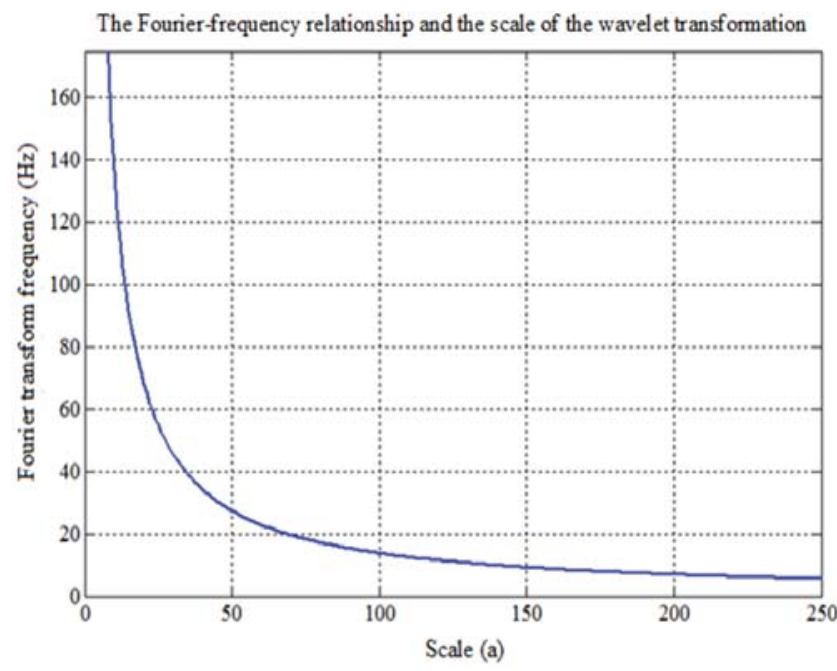

Figure 1. The ratio of the Fourier frequency and the scale of the wavelet transformation

\section{EXPERIMENTAL RESEARCHES}

For carrying out of experimental researches is used the stand shown on a Figure. 2.

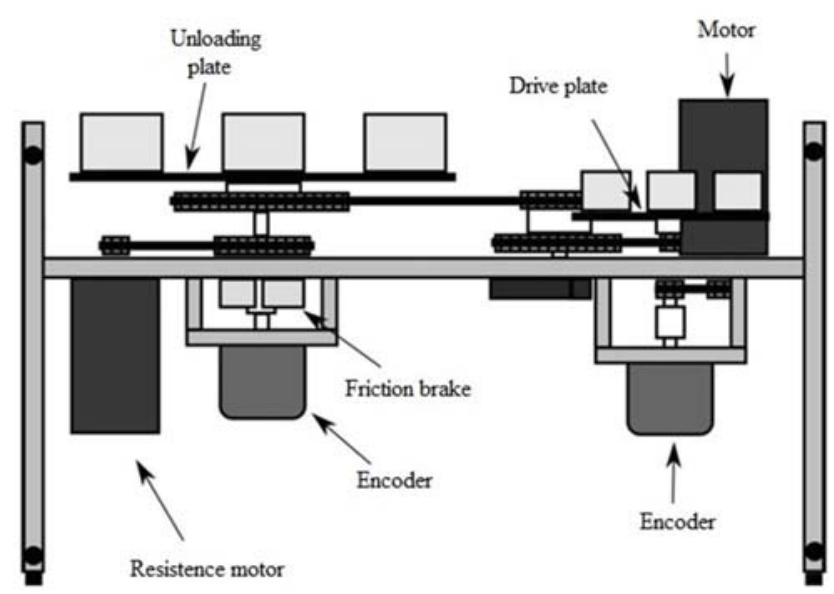

Figure 2. Laboratory stand

The system includes two identical brushless DC motors PITTMAN 5413 (voltage $38.2 \mathrm{~V}$ ), connected to each other by a system of toothed belts (Figure 3).

One engine is the master motor, the second - load motor. To create a resistance to the rotation of the master motor turns on the load motor, which rotates in the opposite direction. The adjustment of the resistance force is possible by changing the speed of rotation of the load motor, and also by installing additional loads on the load plate rigidly connected to the resistance motor shaft through the gear wheel. The encoder is used to determine the position of the motor shaft.

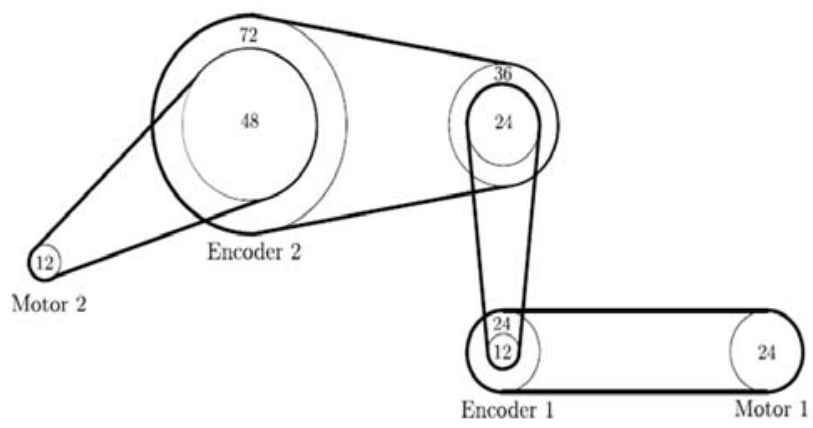

Figure 3. Schematic representation of the mechanical subsystem of the experimental plant

Spectral analysis of the current consumed allows the diagnostics of the electric motor and associated mechanical devices, in which a record of the values of the currents consumed by the electric motor is recorded during a given time interval. The received signal is translated with the help of fast Fourier transforms into the frequency form, the frequencies characteristic for drive failures are allocated and the spectral analysis of the received signal is carried out.

To analyze the state of the drive are taken the current signal and phase voltage of the new faultless drive, which is taken as the reference. The fault tracing is performed at the characteristic frequencies [06] (Table 1) by intelligently comparing the current spectrum with the reference spectrum.

Table 1: Characteristic frequencies of the current signal

\begin{tabular}{|c|l|}
\hline Servo drive faults & \multicolumn{1}{|c|}{ Current signal frequency } \\
\hline Commutation defects & $2 \cdot k \cdot p \cdot f_{r}$ \\
\hline Rotor defects & $2 \cdot p \cdot f_{r}, k \cdot f_{r} \pm 2 \cdot p \cdot f_{r}$ \\
\hline Voltage ripple & $k \cdot f_{s}$ \\
\hline Stator defects & $k \cdot f_{r}$ \\
\hline
\end{tabular}

where $f_{s}$ - frequency of the network supplying the rectifier, $(\mathrm{Hz}) ; f_{r}$ - motor rotor speed, $(\mathrm{Hz}) ; k=1,2,3-$ number of current harmonic ; $p$-the number of poles.

From this table it can be seen that all characteristic frequencies are components of the motor speed or the frequency of the network supplying the rectifier $(50 \mathrm{~Hz})$. The decomposition of the time current signal into a Fourier series and the analysis of the amplitudes at these characteristic frequencies will reveal the object's faults. However, the analysis process is very laborious and will not distinguish the faulty state of the engine from 
a change in its operating mode. Therefore, for further analysis, it is necessary to recalculate the characteristic frequencies of the Fourier analysis into the scale of the wavelet. As a parent function can be chosenany one that meets the requirements for a wavelet. The table 2 shows the result of the conversion to the scale of the Morlet wavelet.

It can be seen from the table that the range of the characteristic wavelet scales is in the range up to 400 , therefore it is expedient to carry out all calculations in the range of scales from 1 to 400 .

Table 2: The ratio of the characteristic frequencies of the Fourier transform and the scale of the wavelet

\begin{tabular}{|c|c|c|}
\hline $\begin{array}{c}\text { Servo drive } \\
\text { faults }\end{array}$ & $\begin{array}{c}\text { Frequencies } \\
\text { of the Fourier } \\
\text { spec-trum }\end{array}$ & $\begin{array}{c}\text { Scale of the } \\
\text { Morlet wavelet }\end{array}$ \\
\hline Commutation & 24 & 48 \\
defects & 48 & 24 \\
& 72 & 16 \\
\hline Rotor defects & 24 & 48 \\
& 27 & 43 \\
& 30 & 39 \\
Voltage ripple & 33 & 35 \\
\hline \multirow{3}{*}{ Stator defects } & 50 & 23 \\
& 100 & 12 \\
\hline
\end{tabular}

Experimental studies were carried out on an motor rotating at a frequency of $3 \mathrm{~Hz}$. In the first experiment, the motor is defective and not loaded, the resistance motor is switched off. In the second experiment, the resistance motor rotates in the opposite direction relative to the main motor at a frequency of $1 \mathrm{~Hz}$, creating phases of the motor, which simulates the short circuit of the stator winding, the load motor is switched off. In the fourth experiment, the engine operates with the fault created, and the load motor rotates from a frequency of $1 \mathrm{~Hz}$ in the direction opposite to the rotation of the motor being examined. The obtained time signals of the current and voltage of the two phases of the motor were decomposed using a Morlet wavelet in the selected range of scales and has been obtained a comparative analysis of the results. The Figure 5 shows the scaling diagrams of the current of the motors in a different technical state.

From the above scaling diagram can be see that the analyzed signal has a peak at motor start-up, and then it goes on an even sinusoid in a range of scales up to 120 . Coefficients for higher scales, characteristic for low motor frequencies are much lower than in this range.
When a load occurs, the scaling is stretched, and the active region has the form of a hyperbola, while higher scales are active in the first seconds of the motor's operation.

The scaling diagram of a faulty unloaded motor has significantly higher wavelet coefficients over the whole range of scales, with an increase in scales characteristic of the main signal from 120 to 220 , and the values of the coefficients at these frequencies are lower than at higher scales, hence, the signal of the faulty motor has more low frequency, than serviceable.

The scaling diagram of a faulty loaded motor has high wavelet coefficients over the whole range of analysis, however, the basic signal pattern also has the form of a hyperbola, which is more stretched to the scale of the wavelet than the serviceable loaded motor. Wavelet coefficients showing the localization of the main signal are lower than on uncharacteristic scales.

The obtained form of the scaling diagrams for the current of the effective phase, as well as the voltages of both phases is similar. Therefore, this type of scaling diagrams is characteristic and allows to determine the technical state of the motor. However, the purpose of the diagnosis is to identify the cause of the failure of the object. To solve this problem will allow analysis of wavelet coefficients on characteristic scales (Table 2). On the Figure 6 is showed the time dependence of the wavelet coefficients of the current signal on the scale that typical for stator malfunction.

From the provided schedules it is visible that the wavelet coefficients of a serviceable unloaded engine have insignificant fluctuations at motor start-up and then the process is practically linearized. When a load appears, the oscillatory process at the start of the motor is more pronounced, but then decreases, however it has features with a certain periodicity to repeat after a certain time interval. Nevertheless, the process is stable, there is no significant increase in the amplitude of the oscillations with time.

The coefficients of the wavelet transform of the faulty motor are much lower than those of the serviceable one and have constant oscillations, which increase when the load appears.

The time dependence of the wavelet coefficients on the characteristic scale for the serviceable phase "B" is shown in Figure 7 , from which it can be seen that the dependence is similar.

When closing rounds of a winding of the stator there is an essential change of amplitude of current that is visible by comparison of wavelet coefficients. At the same time voltage variation on amplitude is practically absent therefore it is logical to assume that the range of changes wavelet coefficients will be not the so considerable, and, therefore, wavelet expansion of a signal of tension will allow to identify an object status more precisely. 


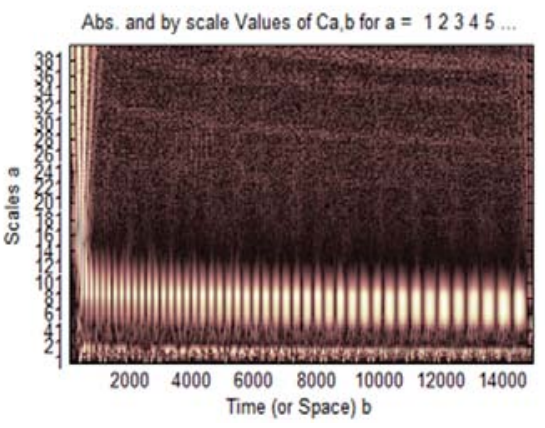

a)

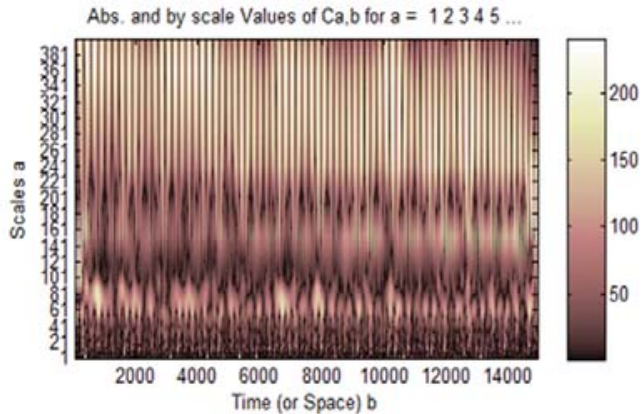

c)
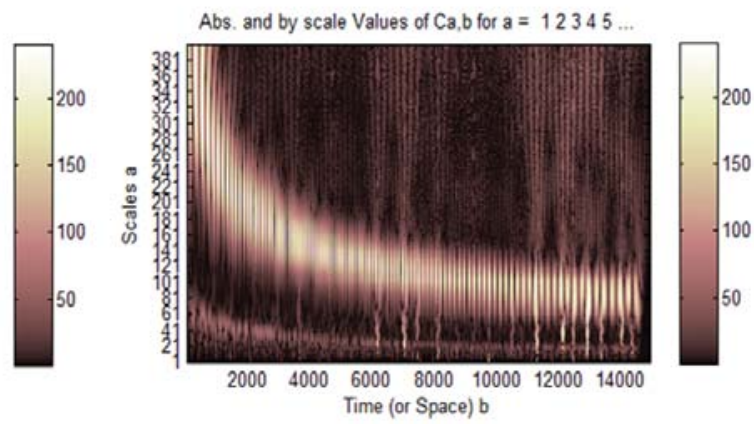

b)

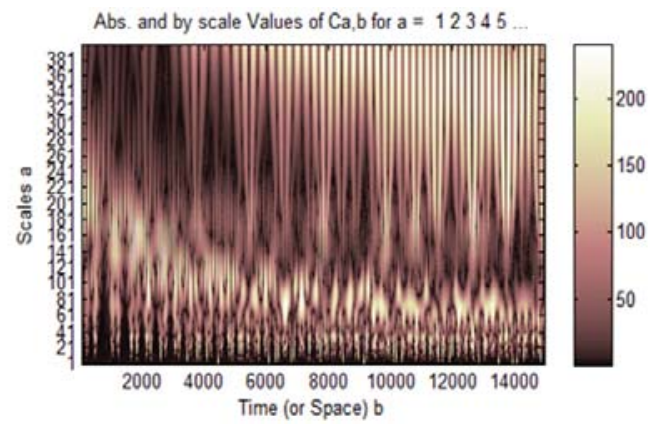

d)

Figure 5. Scalegramms of motors: (a) serviceable without load, (b) serviceable with load, (c) defective without load, (d) defective with load
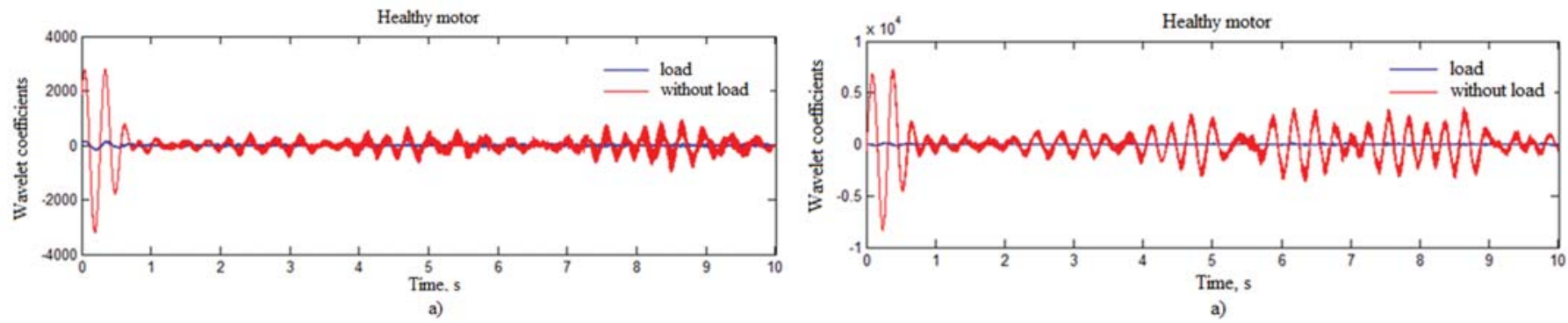

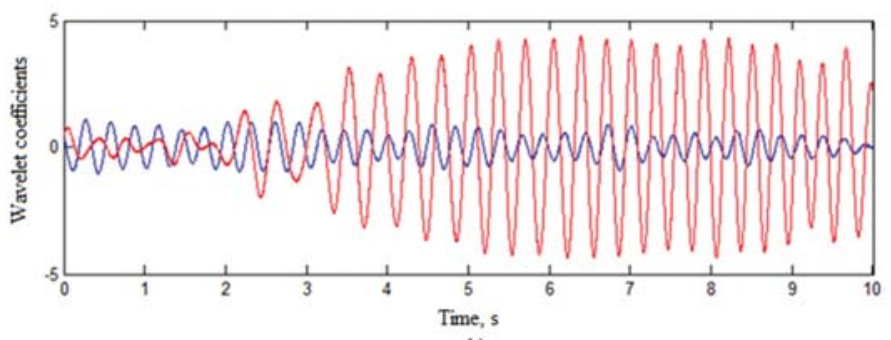

b)

Figure 6: Wavelet stator current factors "A" at scale 386, that typical for stator malfunction:

(a) motor in good condition, (b) faulty motor

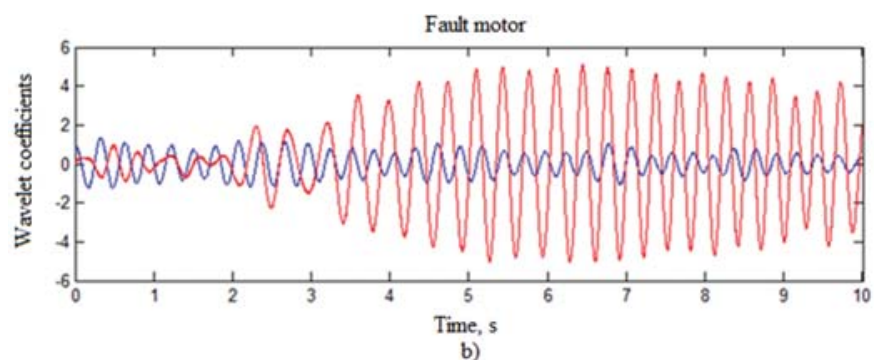

Figure 7: The wavelet coefficients of stator current of phase "B" at scale 386, which characteristic for stator malfunction:

(a) motor in good condition, (b) faulty motor 
Results are given in Figures 8 and 9 wavelet expansions of tension at a characteristic scale.

From these diagrams is visible that the dependence revealed for current remains, however amplitudes of signals of tension of a phase "A" of operational and faulty engines are in one range while tension of a phase " $\mathrm{B}$ " is twice higher that characterizes the entered failure "closing of rounds of a winding of the stator of a phase "A"'.

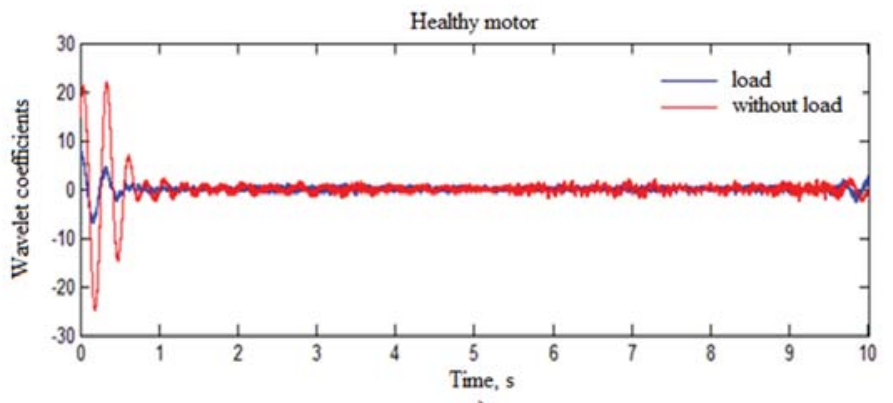

a)

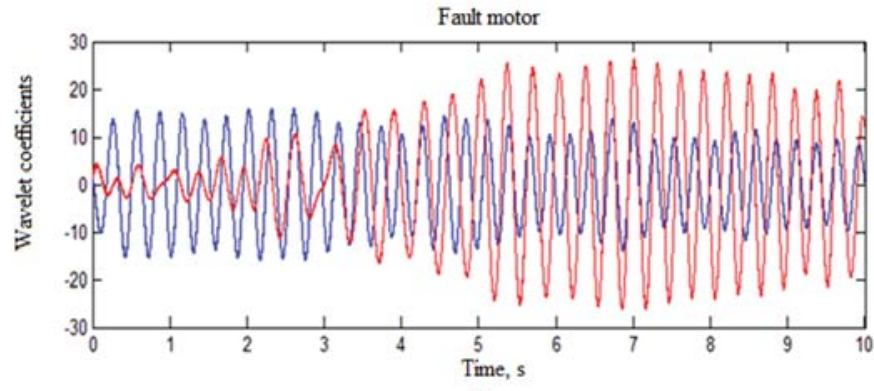

b)

Figure 8: The wavelet coefficients of the feeding tension a phase "A" at a scale of 386 characteristic of malfunction of the stator: (a) serviceable motor, (b) faulty motor

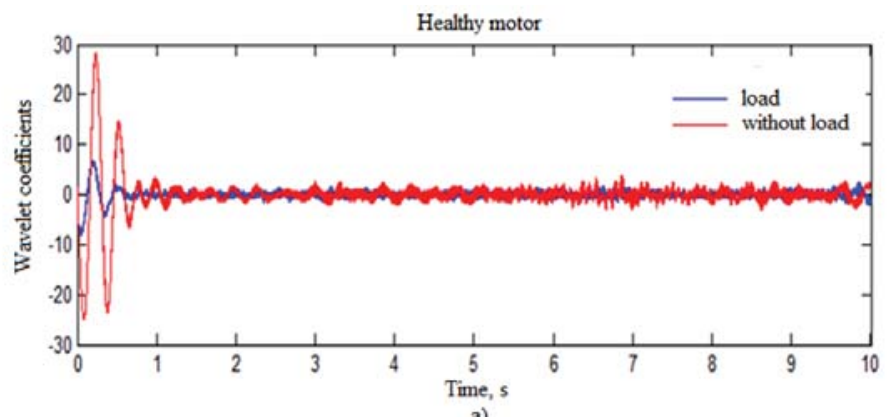

(a)

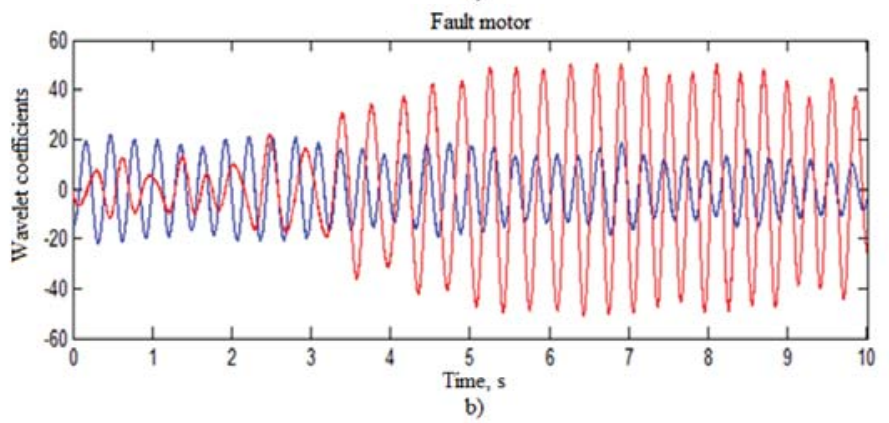

Figure 9: The wavelet coefficients of the feeding tension a phase "B" at a scale of 386 charac-teristic of malfunction of the stator: (a) serviceable motor, (b) faulty motor
The carried-out analysis shows what wavelet values coefficients of current and tension at the scales corresponding to malfunction has an identical characteristic appearance that can be used for intellectual diagnosing of engines. Wavelet values coefficients at the scales not characteristic of the entered malfunction has the appearance given on Figure 10.

From this diagram it is visible, the signal has high density and wavelet small values coefficients, at the same time the signal is regular and completely repeats with the given frequency of following. This type of a signal is characteristic to all frequencies not characteristic the selected failure in independence of technical condition of the motor.

Thus, on analysis results we receive five characteristic signals for diagnosing. Signals of characteristic frequencies for the operational off-load and loaded engines (Figure 9, a), and also an unrepresentative signal (Figure 10), it is expedient to carry to a class "is serviceable" while faulty loaded and off-load to a class "is faulty". This information can be used for simulation of a neural network of classification of technical condition of the engine.
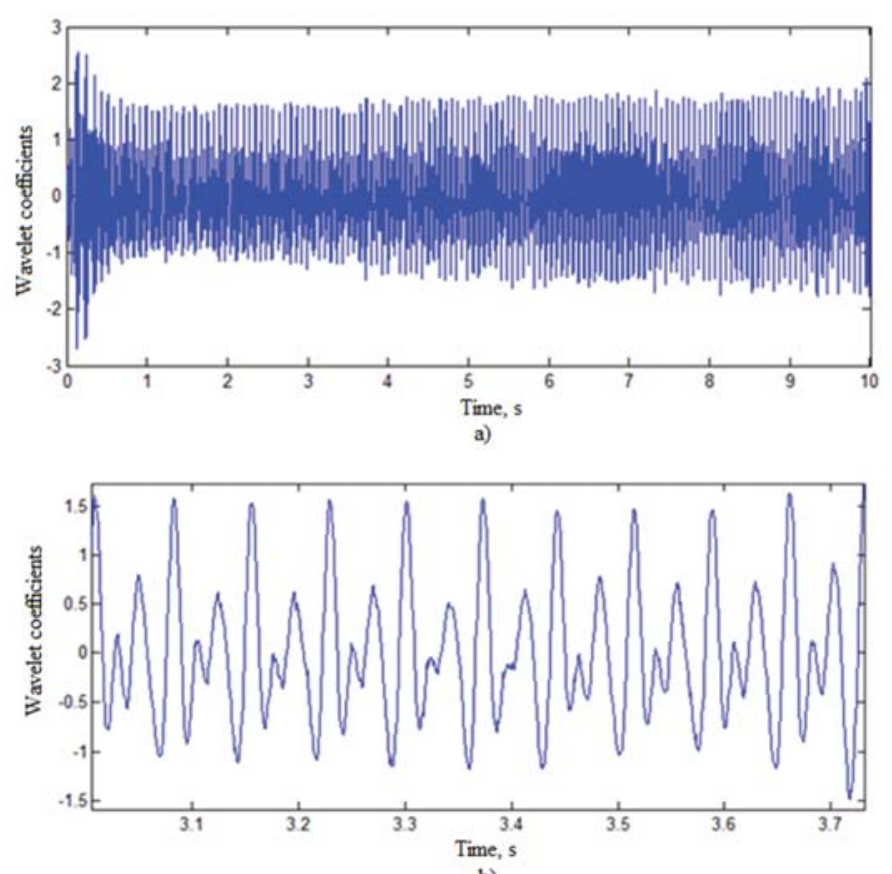

Figure 10: The wavelet coefficients of the feeding tension of the service able and faulty engine at an ucharacteristic scale:

(a) a real signal, (b) in the increased scale 


\section{NEURAL NETWORK MODEL OF CLASSIFICATION OF TECHNICAL CONDITION OF THE ELECTRIC MOTOR UNDER VARIOUS SERVICE CONDITIONS}

For automatic detection of technical condition of the electric drive without involvement of the specialist expert expediently develop a neural network of classification [07-10]. As basic data values are used wavelet coefficients at a scale (Figure 9), characteristic of malfunction, and an uncharacteristic signal (Figure 10). As an entrance is set the matrix containing five lines of characteristic signals. An exit of network is the diagnosis class: "1" - an object is serviceable, "2" - an object is faulty.

For classification of technical condition of an object is modelled the neural network of direct signal transmission having the structure given on Figure 11.

The network contains two layers: hidden and output. The hidden layer has five neurons with tangential function of activation, output - one linear neuron.

For training of neural network is used the Levenberg-Markvardt [11] algorithm intended for optimization of parameters of nonlinear regression models. The algorithm consists in consecutive approach of the set initial values of parameters to a required local optimum.

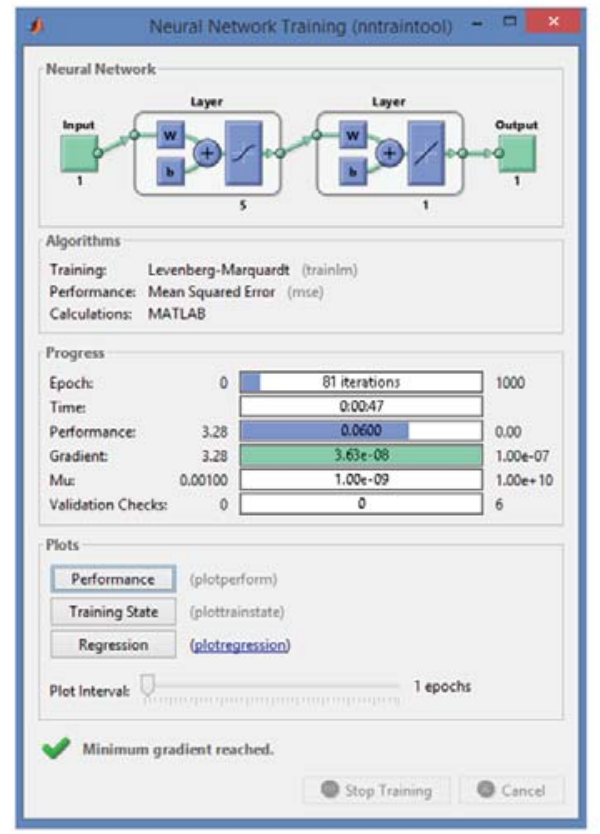

Figure 11. Structure of neural network of classification of technical condition of the electric drive

A teaching selection is set which is many couples of the free variable of $x \in X^{M}$ (network inputs) and dependent variable of $y \in Y^{M}$ (a target vector). The functional dependence is set that represente a regression $y=f\left(\omega, x \_n\right)$ continuously differentiable in the field of $W \times X$. The parameter $\omega$ is a vector of weight factors.

It is required to find such value of a vector $\omega$ which would deliver a local minimum of function of an error:

$$
E_{D}=\sum_{n=1}^{N}\left(y_{n}-f\left(\omega, x_{n}\right)\right)^{2}
$$

Before work of an algorithm is set the initial vector of weight coefficients $\omega$.

1) On each step of iteration is replaced this vector with a vector $\omega+\Delta \omega$. For an increment assessment of $\Delta \omega$ is used the linear approach of function:

$$
f\left(\omega+\Delta \omega, x_{n}\right) \approx f(\omega, x)+J \Delta \omega,
$$

where $J$-Yakobian of function $f\left(\omega, x_{n}\right)$ in a point $\omega$. The matrix can be presented visually in the form:

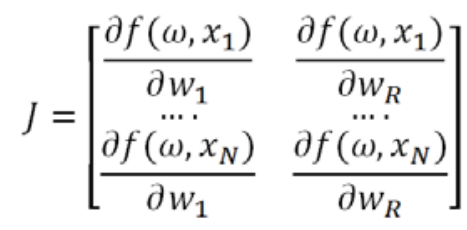

where $\omega=\left[\omega_{1}, \ldots . \omega_{R}\right]^{T}$ is the vector of parameters.

The increment $\Delta \omega$ in a point $\omega$, the delivering minimum of $E_{D}$, is equal to zero therefore for finding of the subsequent value of an increment $\Delta \omega$ we will equate to zero a vector of private derivative $E_{D}$ on weight $\omega$. For this purpose we will present (1) in the form

$$
E_{D}=|y-f(\omega+\Delta \omega)|^{2},
$$

where:

$$
\begin{aligned}
& y=\left[y_{1}, \ldots, y_{N}\right]^{T} \text { and } \\
& f(\omega+\Delta \omega)=\left[f\left(\omega+\Delta \omega, x_{1}\right), \ldots f\left(\omega+\Delta \omega, x_{N}\right)\right]^{T} .
\end{aligned}
$$

Transforming and differentiating this equation:

$$
\begin{aligned}
& |y-f(\omega+\Delta \omega)|^{2}=(y-f(\omega+\Delta \omega))^{T} y- \\
& -f(\omega+\Delta \omega))=f^{T}(\omega+\Delta \omega) f(\omega)- \\
& \left.-2 y^{T} f(\omega+\Delta \omega)\right)+y^{T} y,
\end{aligned}
$$

we will receive:

$$
\frac{\partial E_{D}}{\partial \omega}=\left(J^{T} J\right) \Delta \omega-J^{T}(y-f(\omega)=0
$$

So that to find value $\Delta \omega$ it is necessary to solve system of the linear equations $\Delta \omega=\left(J^{T} J\right)^{-1} J^{T}(y-f(\omega))$.

As the number of conditionality of a matrix of $J^{T} J$ is a square of number of conditionality of a matrix of $J$, a matrix $J^{T} J$ can be degenerate. Therefore, Markvard is entered regularization parameter $\lambda \geq 0$.

2) $\Delta \omega=\left(J^{T} I\right)^{-1} J^{T}(y-f(\omega))$, where $I$ - single matrix. This parameter is appointed on each iteration of an algorithm. If value of a mistake of $E_{D}$ decreases quickly, small value $\lambda$ reduces this algorithm to the Gauss-newton algorithm.

3) The algorithm stops in case the increment $\Delta \omega$ in the subsequent iteration is less than preset value or if the vector of weight coefficients delivers $E_{D}$ mistake, smaller the set size or if the number of cycles of training of neural network is exhausted. Value of a vector $\omega$ on the last iteration is considered required. 
For testing of the trained network were given on an input the samples of learning selection in turn and the network carried unmistakably them to the given class. Further, on an input of a neuronet values were given wavelet coefficients at all characteristic scales (Table 2) of the serviceable off-load motor and the network carried all scales to the class "1" - is "serviceable". Then similarly on an input of a network values for the serviceable loaded drive (rotating speed of the motor of resistance, of $1 \mathrm{~Hz}$ ) moved and the network carried it to the first class.

The analysis faulty off-load and faulty loaded (rotating speed of the motor of resistance of $1 \mathrm{~Hz}$ ) showed what scales 386, 193 and 129 wavelet expansions belong to the class "2" - is "faulty", and all remaining, to the class "1" - is "serviceable" that demonstrates existence of failure of the stator.

Similar tests were executed on the serviceable and faulty motor in case of rotating speed of the motor of resistance of $0,2 \mathrm{~Hz}, 0,4 \mathrm{~Hz}, 0,6 \mathrm{~Hz}$ and $0.8 \mathrm{~Hz}$. In all cases defined the neural network correctly an object status.

Further the similar experiment was made for Daubechies wavelet of 10 order about, Haar, the Mexican hat and Gauss. Recalculation of frequencies of Fourier of conversion to scales of these wavelet is executed. Substitution wavelet coefficients on data of characteristic scales in the trained neural network allowed to identify failure of an object unmistakably. It testifies to correctness of theoretical calculations and adequacy to the developed neural network model of diagnosing.

\section{CONCLUSIONS}

The intellectual method of diagnosing of the electric drive allowing with a high precision to find malfunction of an object is offered, having distinguished her from change of loading. For realization of this method is offered the sharing of wavelet transformation and neural networks. It is established that for realization of this method can be used the parent wavelet. The validity of the theoretical calculations and adequacy of model are confirmed in the large volume of pilot studies.

\section{REFERENCES}

1. T.W. Körner. Fourier Analysis. - Cambridge University Press, 1988.

2. Daubechies. The wavelet transform time-frequency localization and signal analysis. IEEE Trans. Inform. Theory, 1992,pp. 961-1004.

3. T.N. Kruglova. Intelligent Diagnosis of the Electrical Equipment Technical Condition // Procedia Engineering, 2015, Vol 129, pp. 219-224.

4. Peter W. Tsea, Wen-xian Yangb, H.Y. Tama. Machine fault diagnosis through an effective exact wavelet analysis // Journal of Sound and Vibration 277, 2004, pp. 1005-1024.
5. B. Liu, S.F. Ling.On the selection of informative wavelets for machinery diagnosis // Mechanical Systems and Signal Processing 13 (1), 1999, pp. 145-162.

6. Subhasis Nandi, Hamid A. Toliyat, and Xiaodong Li.Condition Monitoring and Fault Diagnosis of Electrical Motors - A Review, IEEE Conference Transactions on energy conversion, Vancouver, Canada // Journals \& Magazines Vol. 20 (4), December 2005, pp. 719-729.

7. Yoon-Seok Lee, Kyung-Tae Kim, Jin Hur.Finite-Element Analysis of the Demagnetization of IPM-Type BLDC Motor With Stator Turn Fault // IEEE transactions on magnetics, vol. 50 (2), pp 70220047022004 .

8. M. Shakouhi, M. Mohamadian, E. Afjei Fault. Tolerant Control of Brushless DC Motors Under Static Rotor Eccentricity // IEEE transactions on industrial electronics, vol. 62 (3), 2015 pp. 1400-1409.

9. Z.K. Penga, Peter W. Tsea, F.L. Chub A comparison study of improved Hilbert-Huang transform and wavelet transform: Application to fault diagnosis for rolling bearing // Mechanical Systems and Signal Processing 19, 2005, pp. 974-988.

10. M.A. Awadallah and M.M. Morcos Diagnosis of Stator Short Circuits in Brushless DC Motors by Monitoring Phase Voltages // IEEE Transactions on Energy Conversion, 2005, vol. 20 (1), pp. 2460-247.

11. T.J. Andersen and B.M. Wilamowski. A modified regression algorithm for fast one layer neural network training // World Congress of Neural Networks, vol. 1, pp. 687-690, Washington, DC, July 17-21, 1995
Paper submitted: 26.07.2017.

Paper accepted: 23.10.2017.

This is an open access article distributed under the CC BY-NC-ND 4.0 terms and conditions. 